\title{
Modulation of SERCA2a expression and function by ultrasound-guided myocardial gene transfection
}

\author{
ZHONGSHAN GOU $^{1 *}$, XINXIN YAN $^{2 *}$, HONGJING JIA ${ }^{1}$, KANGYUN SUN $^{3}$, \\ PING $\mathrm{LI}^{3}$, QIAN ZHANG ${ }^{2}$ and XUEDONG DENG ${ }^{1}$ \\ ${ }^{1}$ Center for Medical Ultrasound; Departments of ${ }^{2}$ Pharmacy and ${ }^{3}$ Cardiology, The Affiliated \\ Suzhou Hospital of Nanjing Medical University, Suzhou, Jiangsu 215000, P.R. China
}

Received May 6, 2019; Accepted March 25, 2020

DOI: $10.3892 / \mathrm{etm} .2020 .9261$

\begin{abstract}
Sarco/endoplasmic reticulum $\mathrm{Ca}^{2+}$-ATPase (SERCA2a) is important for cardiac physiological function and pathological progression. However, intravenous injection, a commonly applied approach for gene delivery in most studies investigating the expression of SERCA2a in cardiomyocytes, has not been particularly satisfactory. Therefore, in the present study, a modified method was used to transfect this gene into the heart. Specifically, a SERCA2a-knockdown lentivirus was directly injected into the myocardium of adult rats under ultrasound guidance, following which the effectiveness and feasibility of this proposed approach were evaluated. The results demonstrated that compared with traditional intravenous injection, the modified gene delivery method resulted in markedly higher transfection efficiency. In addition, the SERCA2a-knockdown rats exhibited higher rates of arrhythmia and weaker ventricular wall motions compared with those in the control rats, with these symptoms more evident in the rats that received a direct injection into the myocardium compared with those that were intravenously injected. These results suggest that ultrasound-guided injection into the myocardium is an efficient and safe method for gene delivery and for inducing the knockdown of SERCA2a protein expression in cardiomyocytes in their native environment.
\end{abstract}

\section{Introduction}

In heart failure (HF), aberrant intracellular calcium $\left(\mathrm{Ca}^{2+}\right)$ homeostasis is associated with systolic and diastolic dysfunction and arrhythmogenesis (1). Although a number of

Correspondence to: Dr Xuedong Deng, Center for Medical Ultrasound, The Affiliated Suzhou Hospital of Nanjing Medical University, 26 Daoqian Street, Suzhou, Jiangsu 215000, P.R. China E-mail: 13962112038@163.com

*Contributed equally

Key words: ultrasound-guided, gene transfection, sarco/endoplasmic reticulum $\mathrm{Ca}^{2+}$-ATPase, heart dysfunction factors are involved in $\mathrm{Ca}^{2+}$-handling abnormalities, alterations in sarco/endoplasmic reticulum $\mathrm{Ca}^{2+}$-dependent ATPase 2a (SERCA2a) appear to serve the most important function (2). SERCA2a is expressed in the cardiac sarcoplasmic reticulum (SR), which serves a central role in intracellular $\mathrm{Ca}^{2+}$ handling by pumping $\mathrm{Ca}^{2+}$ from the cytosol into the SR lumen (3). This enzyme reduces the cytosolic calcium concentration and ensures that an adequate store of calcium is available within the SR for release during the ensuing systole. The expression and activity of SERCA2a significantly decrease during HF onset, which impairs the uptake of $\mathrm{Ca}^{2+}$ into the SR, reduces the subsequent release of $\mathrm{Ca}^{2+}$ from the SR, in turn causing heart dysfunction. Therefore, impaired $\mathrm{Ca}^{2+}$ reuptake resulting from the decreased abundance and reduced activity of SERCA2a is a characteristic of HF (4). SERCA2a-knockout mice have been previously demonstrated to develop systolic and diastolic dysfunction, further supporting the important role of SERCA2a in HF (5). Reduced SERCA2a mRNA expression has been detected in the failing myocardium of humans in addition to animal models $(6,7)$, making the SERCA2a pathway an attractive therapeutic target.

One of the most common methods for investigating the role of a specific protein in the normal heart environment is to transfer small hairpin (sh)RNA or a coding DNA sequence into cultured cardiomyocytes via a plasmid or a viral vector (8-11). However, this approach is accompanied with difficulties that mimic stress factors during the process of myocardial remodeling in vitro (12). In addition, neonatal cardiomyocytes, which are widely used with this method due to their ease of transfection, exhibit physiological and pathological properties that differ from those of adult cells (13). Other approaches, such as intravascular (via the caudal vein) or intraperitoneal systemic injections (14-16), are relatively costly and time consuming, which usually result in low transfection rates and efficacy due to accumulation of the transgene in the bloodstream and nontargeted organs (17-19).

Based on information provided by previous studies (8-11,14-16), the present study attempted to modify the approach for gene delivery to the heart. Specifically, SERCA2a-knockdown lentivirus was directly injected into the myocardium of adult rats under ultrasound guidance. A detailed study was then performed to test the effectiveness and feasibility of this method and to confirm the role of SERCA2a expression in cardiac dysfunction. 


\section{Materials and methods}

Animals. A total of 65 Sprague-Dawley male rats (7-8 weeks old, $250 \mathrm{~g}$; Shanghai Kayon Biological Technology Co., Ltd.) were used. They were kept on a 12 -h light/dark cycle in a temperature-controlled room $\left(22^{\circ} \mathrm{C}\right.$; relative humidity, $\left.45 \%\right)$ with ad libitum access to food and water. The specific criteria used to determine when animals should be euthanized was the end of the experiment or when the ultrasound echocardiography (UCG) and electrocardiograms (ECGs) were finished. The duration of the experiment was 5 days. Animal health and behavior were monitored twice a day, every morning and afternoon. The total number of rats used was 65 , of which 61 were euthanized at the end of the experiment, with 4 rats being found lifeless. The potential causes of mortality for the 4 deceased rats may be toxicity of the lentiviral vector or other infections caused by the experimental procedure. In a previous study by Saliba et al (20), it was suggested that significant transfection occurred in the liver, lungs or other organs, which may be the cause of vector toxicity; in addition, other causes, such as an increase in total collagen in the left ventricle of the heart where the adenoviral vector was injected, have been proposed. However, that previous study used an adenoviral vector, whilst the present study used lentiviral vector. Prendiville et al (21) also reported animal mortality due to this technique and possibly due to complications caused by this experimental procedure.

The present study was approved by the Affiliated Suzhou Hospital of Nanjing Medical University Animal Care and Use Committee (Suzhou, China). Isoflurane was used for anesthetizing the rats. All animal welfare considerations were taken into account, including efforts to minimize suffering and distress, use of anesthetics and special housing conditions. Before the procedure, all animals were intubated and anaesthetized with mechanical ventilation by inhalation of $1-1.5 \%$ isoflurane in $100 \%$ oxygen continuously. Body temperature was maintained by a heating pad. To reduce the possibility of perioperative infection, the procedures were performed in a sterile laminar flow hood, where the virus was delivered aseptically. When the experiment was finished, rats were euthanized intraperitoneally with pentobarbital $(200 \mathrm{mg} / \mathrm{kg})$. The death of rats in the present study was verified by identifying the cessation of breathing and heartbeat by ultrasound.

Ultrasound-guided virus injection. The rats were randomized into four groups as follows: i) Two groups received cardiac injections of either a lentiviral vector for the shRNA-mediated knockdown of SERCA2a $(n=20)$ or the viral vector alone $(n=15)$; ii) one group received the SERCA2a shRNA-knockdown virus via a single-bolus tail-vein injection $(\mathrm{n}=20)$; and iii) the untreated control group, which did not receive any virus $(\mathrm{n}=10)$. The SERCA2a shRNA construct had the following sequence: 5'AAATTCTAACTAGTAAGTCTTTTTTGGAATTAAT-3'. Third generation lentiviral systems were used to generate the pGLV1/U6/GFP vectors (Shanghai GeneChem Co., Ltd.). The anterior wall of the left ventricle (LV) of the rat heart was transfected with the virus, where each rat received two injections $(100 \mu 1 / \mathrm{site})$. The sites of the two injections were located in the middle segment of the anterior wall, which divided the whole anterior wall into basal, middle and apical segments. The viral constructs were diluted with $0.9 \%$ saline to a final concentration of $1 \times 10^{9}$ viral particles in $200 \mu \mathrm{l}$ prior to delivery. Under ultrasound guidance, the virus solution was then injected into the anterior myocardium of the LV as follows.

First, the animals were subjected to anesthesia with isoflurane. The chest hair was removed, following which the rats were placed in a supine position on a platform. Electrode pads were then placed on the rats for continuous ECG monitoring throughout the procedure.

A high-resolution ultrasound system (Vevo 770; VisualSonics, Inc.) coupled with a high-frequency linear array transducer (12-38 MHz) was used. Specifically, it was necessary for the needle and injection area of the myocardium to be shown in the same sonographic view during the operation to ensure that the entire virus delivery process was clearly visualized. The gain, depth and focus of the two-dimensional ultrasound image were adjusted for optimal visualization. Under ultrasound guidance, the needle was introduced intercostally through the chest wall to the cavity of the LV and then withdrawn until its tip reached the target position (one of two sites along the anterior myocardium) in the LV anterior wall over a short period of time $(\sim 20 \mathrm{sec})$. The cardiac injections were visualized on the ultrasound monitor, where all images were documented for analysis.

Determination of transfection efficiency. Vector-GFP-transfected ventricles in the cardiac injection group $(n=4)$ and the tail-vein injection group $(n=4)$ were subjected to examination of GFP labeling. GFP expressed at the site of injection in the anterior myocardium was detected and examined using a Zeiss Axioplan fluorescent microscope (Zeiss AG). GFP expression was detected on day 5 after viral infection. The anterior wall of the heart was sectioned, with slices being taken at a similar position in both groups. Tissues were dehydrated using 30\% sucrose-PBS and embedded with optimal cutting temperature compound, frozen at $-80^{\circ} \mathrm{C}$ and sliced into $15-\mu \mathrm{m}$ sections. Three sections were obtained from each animal and observed under a confocal microscope at a magnification of x200. Six randomly selected fields of view were analyzed for each section. The average percentage of GFP-positive cells within the total cell population was calculated from the sections obtained from each animal. The number of GFP-positive cells relative to the total number of cells was defined as the gene transfection efficiency.

Analysis of LV function by UCG and ECG. M-mode UCG of the rats were acquired using the same ultrasound system 5 days after the cardiac injections. M-mode echocardiogram was performed on the parasternal long-axis section of the LV to record the end-systolic and the corresponding end-diastolic LV anterior wall thickness (LVAWs and LVAWd) and LV inner diastolic and systolic dimensions (LVIDd and LVIDs). The LV ejection fraction (EF) and fraction shortening (FS) were calculated using Visual Sonics analysis software (Vevo770; Visualsonics Inc.). ECG was performed on day 5. A 3-min ECG sample was recorded from each animal for analysis. The ST-T segment and the wide portion of the QRS segment were calculated from the mean curve from the ECG usingLabChart7software (AD Instruments). 

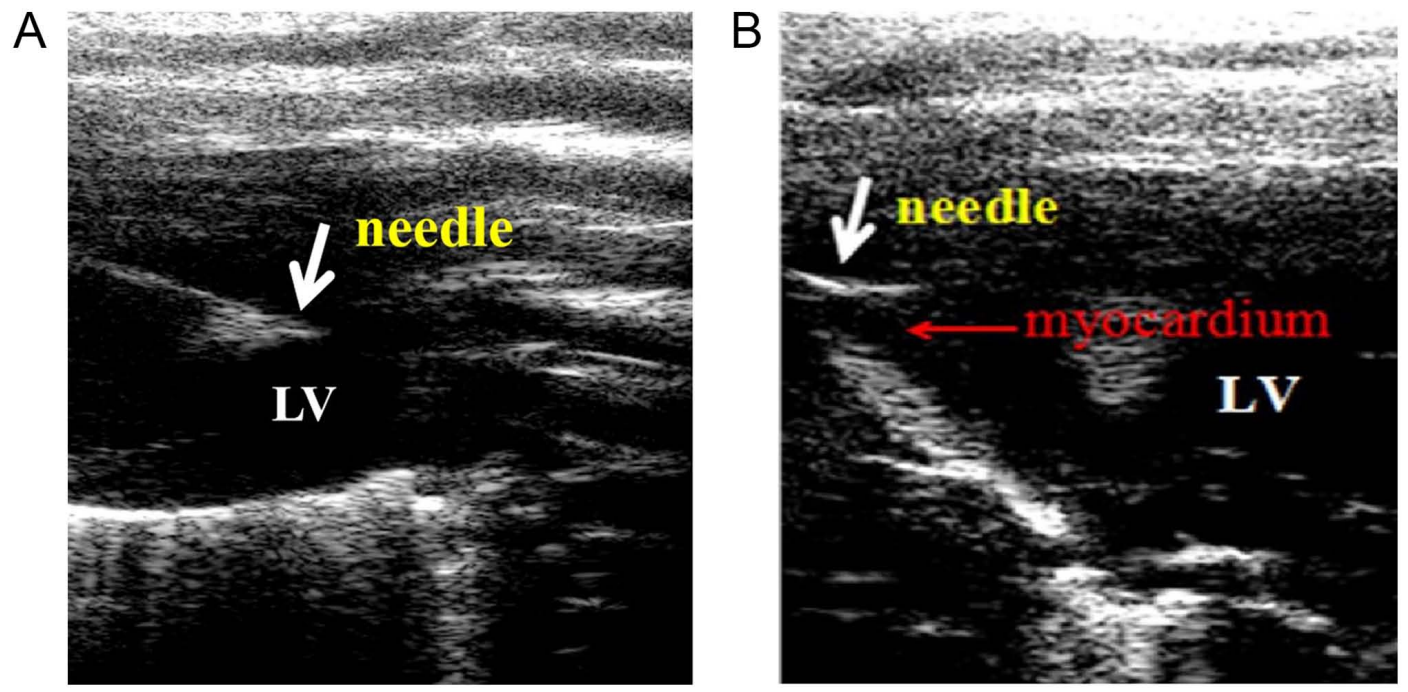

Figure 1. Overview of the ultrasound-facilitated myocardial gene transfection process involving the direct injection of the virus into the anterior wall of the LV. (A) Ultrasound-guided needle puncture into the cavity of the LV (white arrow indicates the needle tip located in the LV cavity). (B) Ultrasound-guided needle withdrawal to the myocardium of the LV (white arrow indicates the needle tip located in the LV myocardium). LV, left ventricle.
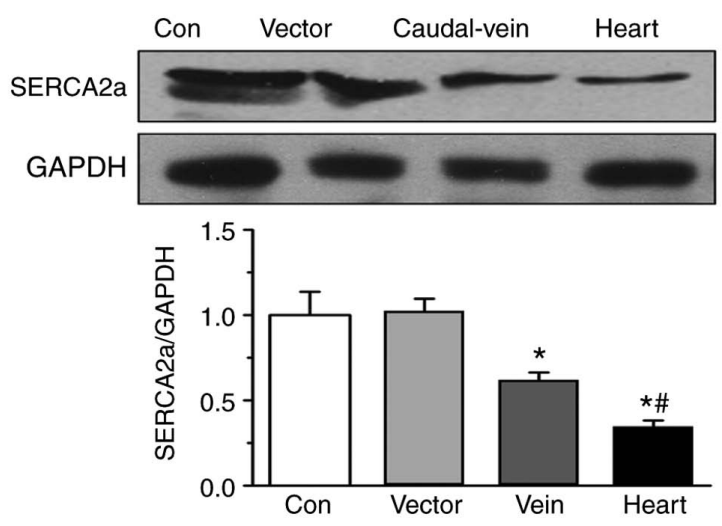

Figure 2. Effects of different methods of shRNA delivery on SERCA2a expression in the heart as measured using western blotting. Cardiomyocytes were transfected with virus containing the empty vector or SERCA2a-knockdown virus using various methods. $\mathrm{n}=4$. ${ }^{*} \mathrm{P}<0.05$ vs. vector, ${ }^{\#} \mathrm{P}<0.05$ vs. vein. $\mathrm{sh}$, small hairpin; SERCA2a, sarco/endoplasmic reticulum $\mathrm{Ca}^{2+}$-ATPase; con, control.

Histology. The hearts were harvested 5 days after the UCG and ECG procedures, fixed in $10 \%$ polyformaldehyde for $24 \mathrm{~h}$ at room temperature, embedded in paraffin, then cut into $10-\mu \mathrm{m}$ sections. Samples were then placed in xylene for 5-10 $\mathrm{min}$, dehydrated in a descending alcohol series $(100,90,80$ and $70 \%)$ and rinsed with distilled water. Sections were then stained with hematoxylin for $5 \mathrm{~min}$ and eosin for 1-3 $\mathrm{min}$ at room temperature. The area of damage was calculated as a percentage of the total area. Three fields of view were selected for one section, with a total of three sections obtained per animal. A mean score was calculated from analysis of animals per group $(n=3)$. All histopathological changes were evaluated in a blinded manner by two investigators (YX and LP). The intercellular space, heart tissue edema and inflammatory infiltration were all assessed under a light microscope (magnification, x400).

Western blotting. Protein was extracted from samples using $1 \%$ PMSF and $1 \%$ protease inhibitor cocktail in RIPA (Santa Cruz
Biotechnology, Inc.). The Pierce BCA assay kit (Thermo Fisher Scientific, Inc.) was used to determine protein concentration and $20 \mu \mathrm{g}$ protein was loaded onto an $8 \%$ SDS-PAGE gel. After transferal to PVDF membranes, 5\% non-fat milk with TBS and $0.1 \%$ Tween-20 was applied at room temperature for $1 \mathrm{~h}$. The following primary antibodies were added to samples and incubated overnight at $4^{\circ} \mathrm{C}$ : Rabbit anti-SERCA2a (cat. no. PA5-78836; 1:1,000; Invitrogen; Thermo Fisher Scientific, Inc.) and mouse anti-GAPDH (cat. no. sc-32233; 1:1,500; Santa Cruz Biotechnology, Inc.). Subsequently, membranes were treated with donkey anti-rabbit DyLight ${ }^{\mathrm{TM}}$ 594-conjugated secondary antibodies (cat. no. SA5-10040; 1:2,000) and goat anti-mouse DyLight ${ }^{\mathrm{TM}}$ 488-conjugated secondary antibodies (cat. no. A32723; 1:5,000; each, Invitrogen; Thermo Fisher Scientific, Inc.) and incubated for $1 \mathrm{~h}$ at room temperature. Samples were then washed with TBS and 0.1\% Tween-20 and visualized with the Super Signal West Pico/Dura chemiluminescent substrate (Pierce; Thermo Fisher Scientific, Inc.).

Statistical analysis. All the experiments were repeated at least three times, and the data are presented as the mean \pm standard deviation. The differences between groups were assessed by post hoc Tukey's test for multiple comparisons. $\mathrm{P}<0.05$ was considered to indicate a statistically significant difference.

\section{Results}

Ultrasound-guided application improved myocardium-specific transfection. The ultrasound-guided cardiac injections resulted in substantial myocardial gene transfection. Fig. 1 illustrates the process used for the injection of SERCA2a-knockdown lentivirus into the heart of the rats using the ultrasound-guided technique. As shown in Fig. S1, enhanced GFP signals for the vector-GFP-transfected ventricles were observed on day 5 , indicating that the viral infection was successful. The number of GFP-positive cells in the cardiac injection group (86\%) was significantly higher compared with that in the tail-vein injection 

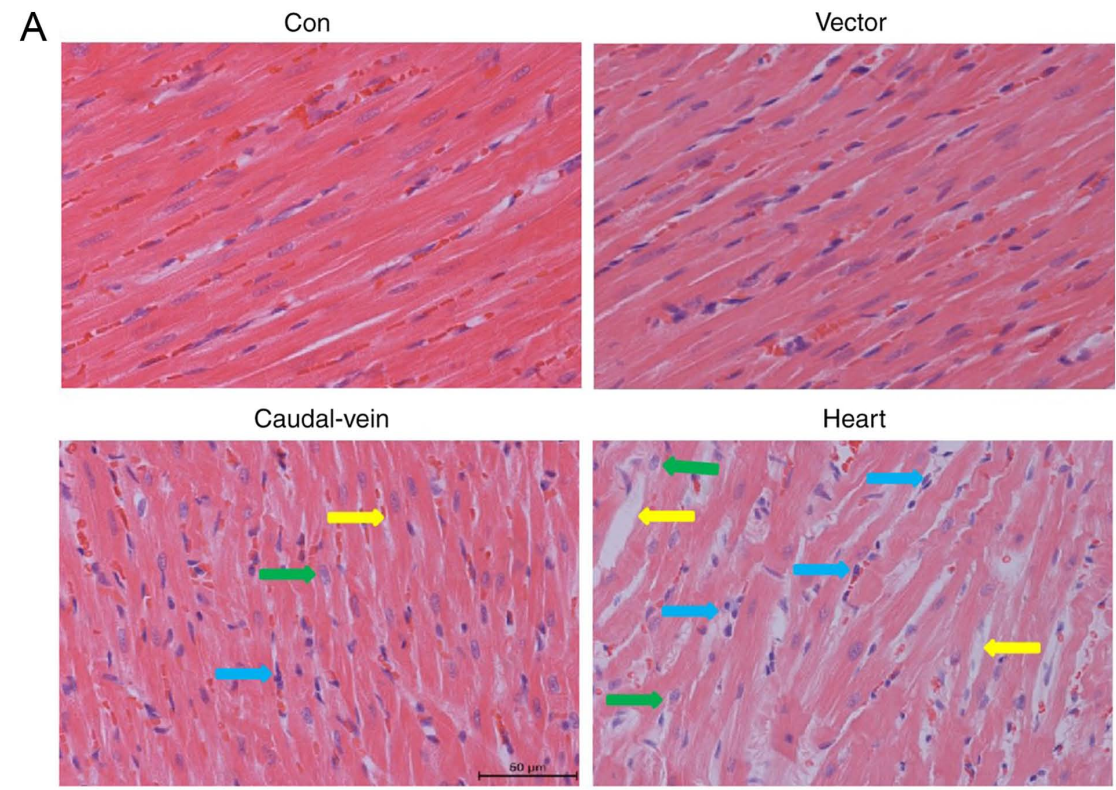

B

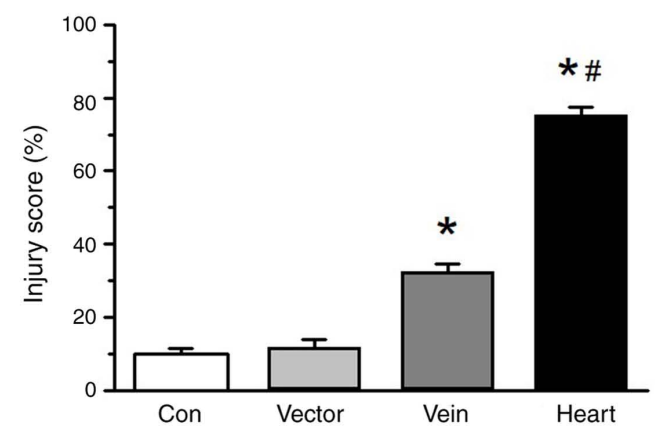

Figure 3. Effects of different methods of SERCA2a-shRNA delivery on heart histology following hematoxylin and eosin staining. (A) Representative images were captured from the groups transfected with virus containing the empty vector or SERCA2a-knockdown virus using the indicated methods (n=6). (B) Heart tissues were allocated injury scores. The yellow, green and blue arrowheads indicate intercellular spaces, heart tissue edema and inflammatory infiltration, respectively. ${ }^{*} \mathrm{P}<0.05$ vs. vector and ${ }^{\#} \mathrm{P}<0.05$ vs. vein. sh, small hairpin; SERCA2a, sarco/endoplasmic reticulum Ca ${ }^{2+}$-ATPase; con, control.

group (48\%; $\mathrm{P}<0.01)$. This finding visually demonstrated that the ultrasound-guided approach is superior compared with intravenous injections. Additionally, the inter-animal variation in the transfection efficiency was found to be low, as shown by the small standard deviation observed in Fig. S1. Western blotting data demonstrated that SERCA2a expression on day 5 was significantly lower in the cardiac injection group compared with that in the tail-vein injection, vector-only and control groups (Fig. 2). This observation suggests that ultrasound-guided application improved myocardium-specific transfection.

SERCA2a knockdown induces cardiomyocyte injury. Hematoxylin and eosin staining results demonstrated a mixture of pathological changes in the mice administered with cardiac injections of the SERCA2a-knockdown virus, including cardiomyocyte hypertrophy, myocardial disarray, intercellular space alterations, heart tissue edema and inflammatory cell infiltration. To a limited extent, similar changes were also evident in the group of mice that received SERCA2a-knockdown virus injections via the tail vein (Fig. 3A). Neither the group that received the injection with the vector alone nor the control group exhibited these types of histopathological changes. The injury score was observed in $>30 \%$ of the myocardium in the hearts of the mice that received injections of the SERCA2a-knockdown virus via the tail vein, whereas $>70 \%$ of the myocardium in the hearts of the mice belonging to the cardiac injection group was injured (Fig. 3B). These data suggest that SERCA2a knockdown induces cardiomyocyte injury.

SERCA2a knockdown induces cardiomyocyte dysfuncton. To confirm the role of SERCA2a in cardiomyocyte dysfunction in vivo, the effects of knocking down SERCA2a levels via the injection of SERCA2a-knockdown virus into the rat heart on the EF and FS of the LV were calculated using UCG. EF and FS were found to be significantly lower in the myocardium transfected with the SERCA2a-knockdown lentivirus compared with those in the myocardium of the rats of the vector and tail-vein injection groups, suggesting that the cardiac injection group exhibited marked deterioration in cardiac function (Fig. 4). In addition, ST-segment elevation, widening of the QRS complex and premature beats were observed in the ECG recordings obtained from the rats belonging to the injection groups, which suggested that reduced SERCA2a expression altered the ventricular conduction properties. Additionally, higher occurrences of ST-T elevation and greater QRS widening were observed in the cardiac injection group compared with those in the tail-vein injection group (Fig. 5). 

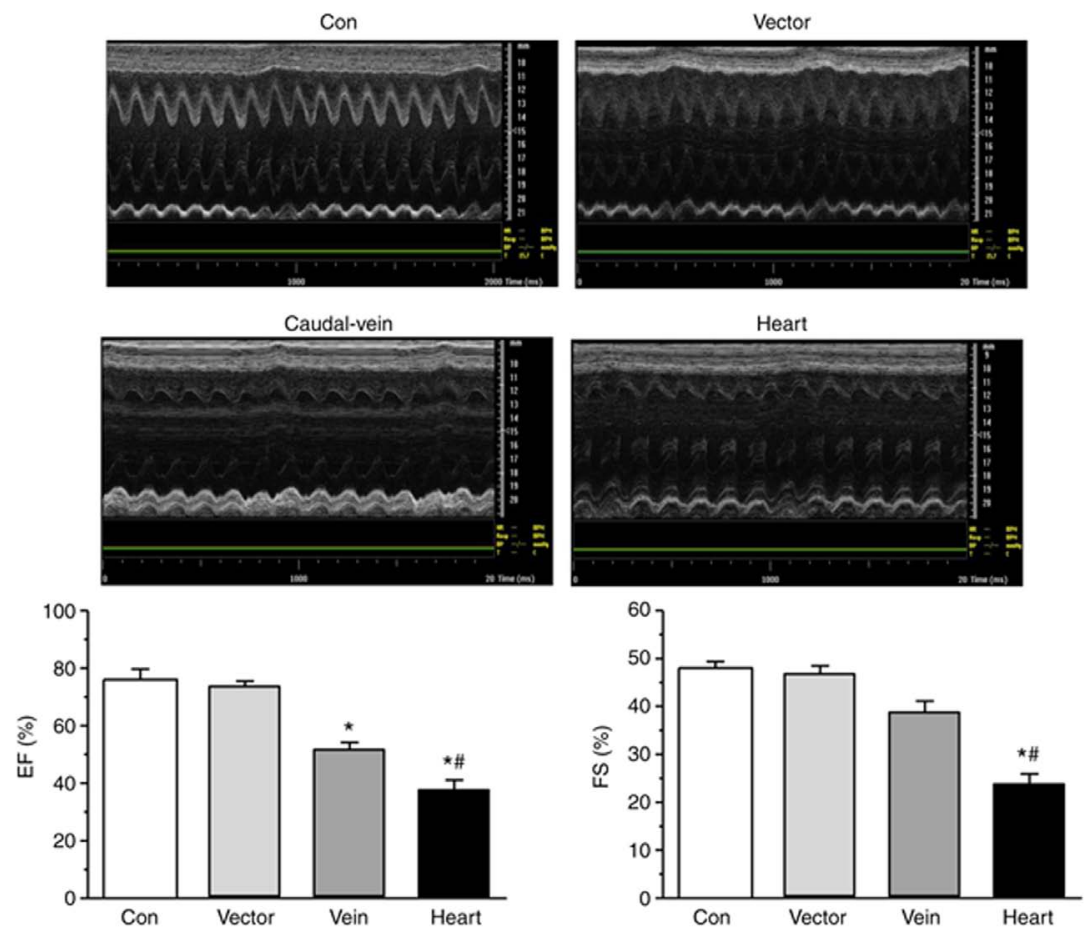

Figure 4. Representative M-mode echocardiography images of the left ventricle obtained 5 days after caudal-vein or heart SERCA2a-shRNA lentiviral injection. The EF and FS were measured from M-mode echocardiographs and quantified ( $\mathrm{n}=8$ ). ${ }^{\mathrm{P}} \mathrm{P}<0.05$ vs. vector and " $\mathrm{P}<0.05 \mathrm{vs}$. vein. EF, ejection fraction; FS, fraction shortening; con, control.
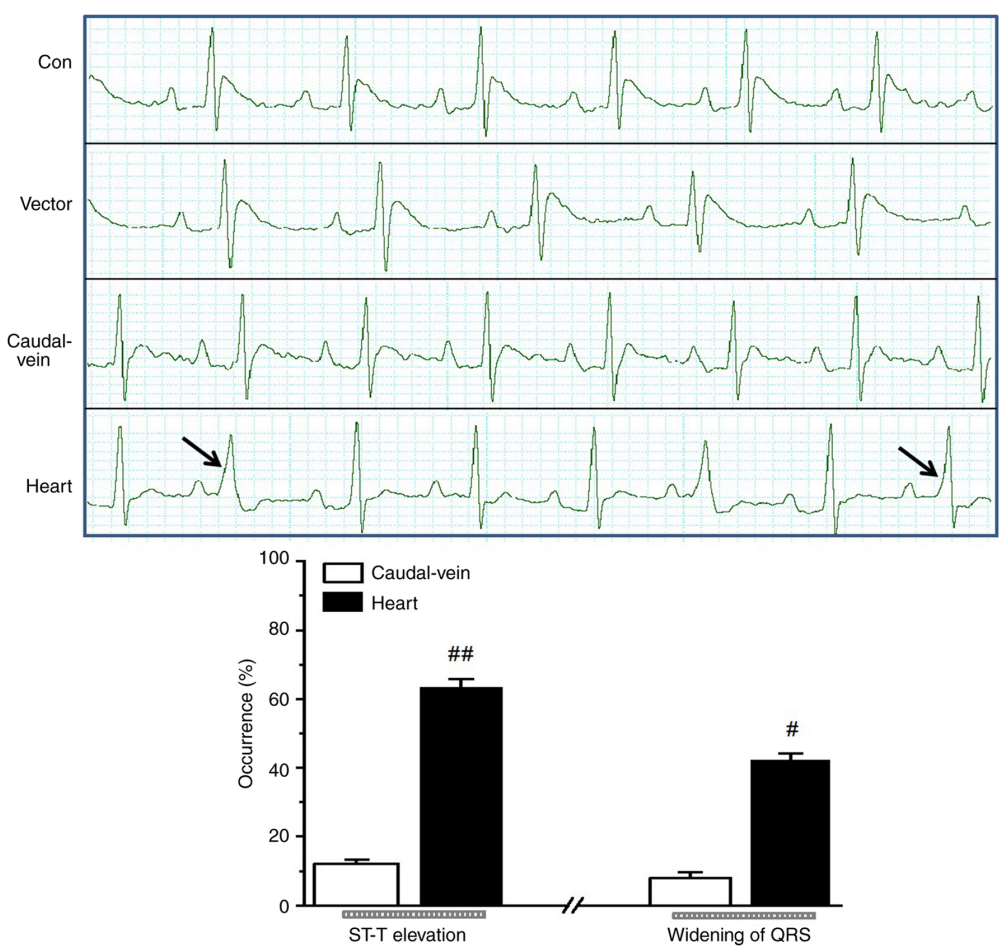

Figure 5. Effects of different methods of SERCA2a-shRNA delivery on electrocardiogram recordings from anesthetized rats obtained 5 days after injection. SERCA2a knockdown by either caudal-vein or heart injection, exhibited abnormal electrocardiograms. Higher ST-T elevation and greater widening of the QRS were observed in the heart injection group $(\mathrm{n}=10)$ compared with those in the caudal-vein injection group ( $\mathrm{n}=10)$. Black arrows indicate $\mathrm{QRS}$ locations. ${ }^{\#} \mathrm{P}<0.05$ and ${ }^{\# \#} \mathrm{P}<0.01$ vs. caudal-vein. sh, small hairpin; SERCA2a, sarco/endoplasmic reticulum $\mathrm{Ca}^{2+}$-ATPase; con, control; ST-T, non-specific ST-T-wave; QRS, QRS complex.

\section{Discussion}

The results of the present study demonstrated that ultrasound-guided cardiac viral injection efficiently induced gene transfer into the heart of the rats, as shown by the observed reduction in SERCA2a expression in cardiomyocytes and 
greater cardiac dysfunction detected in the rats that received cardiac SERCA2a-shRNA injections compared with the rats that received identical injections via the tail-vein $(22,23)$. Previous studies have associated cardiac dysfunction with reduced SERCA2a expression in humans and animals $(24,25)$. Results found in the present study therefore confirmed the pivotal role of SERCA2a in the physiological function of the rat heart.

Ultrasound-guided cardiac injection has a number of benefits for gene transfection. Compared with the caudal vein injection approach, this technique resulted in enhanced, cost-effective, tissue-specific transfection that required lower viral titers. The majority of studies involving the transfection of a gene into the heart have used systemic injection, which is associated with a number of shortcomings, including dilution of the transgene in the circulation and the nonspecific uptake of the transgene by surrounding cells, reducing the expression levels in the heart $(26,27)$. In addition, the number of cells that have been successfully transfected remains difficult to ascertain. The present study found that the efficiency of systemic transfection was markedly lower compared with that by local myocardial-specific transfection via direct cardiac injection. However, immunogenicity and toxicity are important aspects of the technique used in the present study that require investigation in subsequent studies.

Another major advantage of ultrasound-guided cardiac injection is the drastically reduced invasiveness. Saliba et al (20) achieved viral delivery to the heart via open-chest injection. Compared with the approach of Saliba et al (20), ultrasound-guided injection did not induce any significant changes in ventricular function or the ECG findings when comparing control and empty vector groups, where it was possible to perform the real-time monitoring of cardiac function at any time following gene delivery. Therefore, the key processes and order of procedures in the approach presented in the present study differed from those of this previously described technique. The present study describes a feasible and semi-invasive method that avoids open-chest surgery, which is associated with morbidity and mortality (28), thereby allowing the animals to recover more rapidly with minimal inflammatory sequelae. Prendiville et al (21) previously introduced a technique involving ultrasound-guided cardiac injection in mice and demonstrated its advantages. Based on that study, the present study delivered SERCA2a-knockdown lentivirus into rats via ultrasound-guided cardiac injection, the results of which were then with those that received the same lentivirus via tail-vein injection. The resulting SERCA2a protein expression was subsequently assessed, which demonstrated further the function of SERCA2a in the heart.

A potential limitation of the present study is the potential use of a lentiviral vector in patient treatment. The majority of studies into this are still in the early stages, where further basic and clinical research is necessary to verify the long-term safety and efficacy of the application of lentiviral vectors on patients for therapeutic purposes.

In conclusion, the present study improved our previous method for myocardial gene delivery by administering ultrasound-guided minimally-invasive injections directly into the rat heart. This technique provided a safe method for conducting protein functional studies. The present study also investigated and confirmed the critical physiological role of SERCA2a in the myocardium. The simplicity and directness of the proposed approach suggest broader applicability to the study of other proteins.

\section{Acknowledgements}

Not applicable.

\section{Funding}

The present study was supported by a Suzhou Basic Research in Medical and Health Application Grant (grant no. SYSD2016109) and Special Diagnosis Techniques for Clinical Key Diseases of Suzhou Municipal Health and Family Planning Commission (grant no. LCZX201610).

\section{Availability of data and materials}

The datasets used and/or analyzed during the currenty study are available from the corresponding author on reasonable request.

\section{Authors' contributions}

ZG and XY performed the experiments and analyzed the data. ZG performed injections and design the experiments. HJ performed H\&E staining. KS planned and performed western blotting. PL and QZ determined the UCG and ECG. $\mathrm{XD}$ and $\mathrm{XY}$ contributed to the writing the manuscript and analyzing the data. All authors read and approved the final manuscript.

\section{Ethics approval and consent to participate}

The present study was approved by the Affiliated Suzhou Hospital of Nanjing Medical University Animal Care and Use Committee (Suzhou, China).

\section{Patient consent for publication}

Not applicable.

\section{Competing interests}

The authors declare that they have no competing interests.

\section{References}

1. Val-Blasco A, Piedras MJGM, Ruiz-Hurtado G, Suarez N, Prieto P, Gonzalez-Ramos S, Gómez-Hurtado N, Delgado C, Pereira L, Benito G, et al: Role of NOD1 in heart failure progression via regulation of $\mathrm{Ca}^{2+}$ handling. J Am Coll Cardiol 69: 423-433, 2017.

2. Hamilton S and Terentyev D: Altered intracellular calcium homeostasis and arrhythmogenesis in the aged heart. Int $\mathrm{J}$ Mol Sci 20: E2386, 2019.

3. Meyer M, Schillinger W, Pieske B, Holubarsch C, Heilmann C, Posival H, Kuwajima G, Mikoshiba K, Just H and Hasenfuss G: Alterations of sarcoplasmic reticulum proteins in failing human dilated cardiomyopathy. Circulation 92: 778-784, 1995.

4. Marks AR: Calcium cycling proteins and heart failure: Mechanisms and therapeutics. J Clin Invest 123: 46-52, 2013. 
5. Andersson KB, Birkeland JA, Finsen AV, Louch WE, Sjaastad I, Wang Y, Chen J, Molkentin JD, Chien KR, Sejersted OM, et al: Moderate heart dysfunction in mice with inducible cardiomyocyte-specific excision of the Serca2 gene. J Mol Cell Cardiol 47: 180-187, 2009.

6. Chaanine AH, Nonnenmacher M, Kohlbrenner E, Jin D, Kovacic JC, Akar FG, Hajjar RJ and Weber T: Effect of bortezomib on the efficacy of AAV9.SERCA2a treatment to preserve cardiac function in a rat pressure-overload model of heart failure. Gene Ther 21: 379-386, 2014.

7. Kho C, Lee A, Jeong D, Oh JG, Gorski PA, Fish K, Sanchez R, DeVita RJ, Christensen G, Dahl R, et al: Small-molecule activation of SERCA2a SUMOylation for the treatment of heart failure. Nat Commun 6: 7229, 2015.

8. Doroudgar S, Quijada P, Konstandin M, Ilves K, Broughton K, Khalafalla FG, Casillas A, Nguyen K, Gude N, Toko H, et al: S100A4 protects the myocardium against ischemic stress. J Mol Cell Cardiol 100: 54-63, 2016.

9. Isner JM: Myocardial gene therapy. Nature 415: 234-239, 2002.

10. Jiang J, Burgon PG, Wakimoto H, Onoue K, Gorham JM, O'MearaCC,FomovskyG,McConnellBK,LeeRT,SeidmanJG,etal: Cardiac myosin binding protein $\mathrm{C}$ regulates postnatal myocyte cytokinesis. Proc Natl Acad Sci USA 112: 9046-9051, 2015.

11. Bühring HJ, Lang F, Sorg RV, Langer $\mathrm{H}$ and Gawaz M: Activated platelets interfere with recruitment of mesenchymal stem cells to apoptotic cardiac cells via high mobility group box 1/Toll-like receptor 4-mediated Down-regulation of hepatocyte growth factor receptor MET. J BiolChem 289: 11068-11082, 2014.

12. Pironti G, Bersellini-Farinotti A, Agalave NM, Sandor K Fernandez-Zafra T, Jurczak A, Lund LH, Svensson CI and Andersson DC: Cardiomyopathy, oxidative stress and impaired contractility in a rheumatoid arthritis mouse model. Heart 104 2026-2034, 2018.

13. Bardot ES and Dubois NC: A watershed finding for heart regeneration. Cell 176: 947-949, 2019.

14. Fletcher ML, Ogg MC, Lu L, Ogg RJ and Boughter JD Jr: Overlapping representation of primary tastes in a defined region of the gustatory cortex. J Neurosci 37: 7595-7605, 2017.

15. Hardee CL, Arévalo-Soliz LM, Hornstein BD and Zechiedrich L: Advances in Non-Viral DNA Vectors for Gene Therapy. Genes (Basel) 8: 65, 2017.

16. Roostalu U, Aldeiri B, Albertini A, Humphreys N, Simonsen-Jackson M, Wong JKF and Cossu G: Distinct cellular mechanisms underlie smooth muscle turnover in vascular development and repair. Circ Res 122: 267-281, 2018.

17. Bevan AK, Duque S, Foust KD, Morales PR, Braun L, Schmelzer L, Chan CM, McCrate M, Chicoine LG, Coley BD, et al: Systemic gene delivery in large species for targeting spinal cord, brain, and peripheral tissues for pediatric disorders. Mol Ther 19: 1971-1980,2011.
18. Bruegmann T, van Bremen T, Vogt CC, Send T, Fleischmann BK and Sasse P: Optogenetic control of contractile function in skeletal muscle. Nat Commun 6: 7153, 2015.

19. von Burstin J, Diersch S, Schneider G, Reichert M, Rustgi AK and Schmid RM: Detection of tumor suppressor genes in cancer development by a novel shRNA-based method. Mol Cancer Res 13: 863-869, 2015.

20. Saliba Y, Mougenot N, Jacquet A, Atassi F, Hatem S, Farès N and Lompré AM: A new method of ultrasonic nonviral gene delivery to the adult myocardium. J Mol Cell Cardiol 53: 801-808, 2012.

21. Prendiville TW, Ma Q, Lin Z, Zhou P, He A and Pu WT: Ultrasound-guided transthoracic intramyocardial injection in mice. J Vis Exp 90: e51566, 2014.

22. Hadri L, Kratlian RG, Benard L, Maron BA, Dorfmüller P, Ladage D, Guignabert C, Ishikawa $\mathrm{K}$, Aguero J, Ibanez B, et al: Therapeutic efficacy of AAV1.SERCA2a in monocrotaline-induced pulmonary arterial hypertension. Circulation 128: 512-523, 2013.

23. Verbist KC, Field MB and Klonowski KD: Cutting edge: IL-15-independent maintenance of mucosally generated memory CD8 T cells. J Immunol 186: 6667-6671, 2011.

24. Kronenbitter A, Funk F, Hackert K, Gorreßen S, Glaser D, Boknik P, Poschmann G, Stühler K, Isić M, Krüger M, et al: Impaired $\mathrm{Ca}^{2+}$ cycling of nonischemic myocytes contributes to sarcomere dysfunction early after myocardial infarction. J Mol Cell Cardiol 119: 28-39, 2018

25. Zsebo K, Yaroshinsky A, Rudy JJ, Wagner K, Greenberg B, Jessup $M$ and Hajjar RJ: Long-term effects of AAV1/SERCA2a gene transfer in patients with severe heart failure: Analysis of recurrent cardiovascular events and mortality. Circ Res 114: 101-108, 2014.

26. Mays LE, Wang L, Lin J, Bell P, Crawford A, Wherry EJ and Wilson JM: AAV8 induces tolerance in murine muscle as a result of poor APC transduction, T cell exhaustion, and minimal MHCI upregulation on target cells. Mol Ther 22: 28-41, 2014.

27. Shao CH, Capek HL, Patel KP, Wang M, Tang K, DeSouza C, Nagai R, Mayhan W,Periasamy M and Bidasee KR:Carbonylation contributes to SERCA2a activity loss and diastolic dysfunction in a rat model of type 1 diabetes. Diabetes 60: 947-959, 2011.

28. Parsa CJ, Reed RC, Walton GB, Pascal LS, Thompson RB, Petrofski JA, Emani SM, Folgar F, Riel RU, Nicchitta CV, et al: Catheter-mediated subselective intracoronary gene delivery to the rabbit heart: Introduction of a novel method. J Gene Med 7: 595-603, 2005.

This work is licensed under a Creative Commons Attribution-NonCommercial-NoDerivatives 4.0 International (CC BY-NC-ND 4.0) License. 